\title{
HANNIBAL AD PORTAS, O LOS FUTUROS PERFILES PROFESIONALES DE LA INFORMACIÓN
}

\author{
Hannibal ad portas, or the future of information \\ professional proffles
}

\section{Jesús Tramullas}

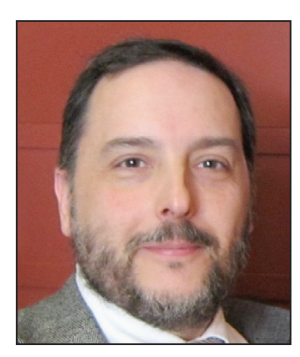

Jesús Tramullas es profesor titular en el Departamento de Ciencias de la Documentación de la Universidad de Zaragoza. Socio senior de ACM, ISKO y Sedic. Miembro de Wikimedia.es y Drupal Association. Investigador principal de proyectos de investigación financiados sobre web semántica, web social y servicios de información digital. Sus líneas de investigación se centran en el diseño e implementación de servicios de información digital, gestión de contenidos, comportamiento informacional, bibliotecas digitales y herramientas de software libre para la gestión de información. http://orcid.org/0000-0002-5374-9993

Universidad de Zaragoza, Departamento de Ciencias de la Documentación Pedro Cerbuna, 12. 50009 Zaragoza, España http://tramullas.com tramullas@unizar.es

\section{Resumen}

La identificación de perfiles profesionales se contempla como necesaria para adecuar la formación de los estudiantes y el desarrollo profesional posterior. Se realiza una revisión bibliográfica selectiva sobre la identificación de perfiles profesionales durante las dos últimas décadas. Se concluye que debe atenderse prioritariamente a la definición y adquisición de competencias, que permitan el desarrollo profesional en entornos caracterizados por cambios dinámicos y continuos.

\section{Palabras clave}

Perfiles profesionales; Desarrollo profesional; Competencias en información; Información y documentación; Biblioteconomía; Profesionales de la información.

\begin{abstract}
The identification of information professional profiles is necessary in order to develop studies for the current labor market and to improve professional development for students. A literature review was carried out on selected papers published within the last two decades. Findings indicate that faculty must address the definition and acquisition of skills to prepare students for a dynamic and constantly changing professional environment.
\end{abstract}

\section{Keywords}

Professional profiles; Professional development; Information skills; Information science; Librarianship; Information professionals.

Tramullas, Jesús (2016). "Hannibal ad portas, o los futuros perfiles profesionales de la información". El profesional de la información, v. 25, n. 2, pp. 157-162.

http://dx.doi.org/10.3145/epi.2016.mar.01

\section{El enemigo a las puertas}

Tito Livio, en Ab urbe condita, narra el temor de los habitantes de Roma al ver aparecer a Aníbal Barca y a su ejército ante sus murallas, tras la batalla de Cannas (216 a. C). Hannibal ad portas se convirtió en la frase que los progenitores romanos usaban para asustar a sus hijos ante un peligro grave e inminente. En la actualidad se podría pensar que las ciencias de la información y la documentación (en adelante Information Science, o IS, adoptando la terminología académica internacional) se encuentran ante una situación similar: numerosos enemigos acampan a sus puertas, amenazando con ocupar su hábitat natural, y parecen ser pocas y débiles las defensas tras las que refugiarse. En una socie- 
dad cada vez más mediada tecnológicamente, los tiempos se acortan progresivamente. En paralelo, la necesidad de información especializada gestionada con herramientas específicas a través de servicios y productos adaptados a las necesidades se configura como un área estratégica. La información y el conocimiento son las características dominantes en la sociedad, y cualquier actividad humana tiene que tratar, en todos los sentidos, con ellos. Se trata de un entorno muy volátil, dinámico, de cambio rápido y continuo desarrollo profesional.

Una de las medidas defensivas que parece oportuno adoptar es la identificación o búsqueda de perfiles profesionales mejor adaptados a un entorno cambiante y dinámico, que pongan en valor las habilidades y capacidades de los profesionales. Los desafíos planteados parecen exigir un proceso de "adopción acelerada de la modernidad". Sin embargo, un peligro evidente en la definición de perfiles profesionales es el que plantea la adopción de buzzwords, generalmente anglosajonas, que pretenden definir y establecer prometedores campos de actividad profesional. Por lo común estas novedades corresponden a modas pasajeras, y su adopción no está apoyada en una reflexión seria y sistemática sobre el concepto que se trate y su contenido. Con la misma velocidad con la que llegan tienden a desaparecer, y suelen ser sustituidas por otra nueva modernidad. Esta volatilidad de nociones, atribuciones y características delinea un paisaje nebuloso y poco definido, en el cual resulta difícil encontrar puntos de referencia que sirvan para planificar una actividad profesional, y por ende dificulta alcanzar un reconocimiento social estable, muy demandado por otra parte por los académicos y profesionales.

Un peligro evidente en la definición de perfiles profesionales es la adopción de buzzwords que pretenden definir y establecer prometedores campos de actividad profesional

\section{Vigilando al enemigo}

La realización de estudios de prospectiva siempre es complicada. Las perspectivas e indicadores que parecen señalar en una dirección siempre pueden verse afectados por factores imprevistos, o cambios radicales en los contextos sociales, económicos y culturales, que acaben dando como resultado situaciones diferentes a las que en un primer momento parecían evidentes. Basta ver en qué ha quedado la tan traída y llevada "generación Google", que ha resultado ser un estereotipo ampliamente sobrestimado, y no ha llegado a alcanzar las habilidades que se le presuponían (Rowlands et al., 2008), ni ha demostrado tener las competencias informacionales básicas que se esperaban.

Esto no quiere decir que no sea necesario e incluso perentorio revisar el panorama de los perfiles profesionales presentes y futuros para los estudiantes y profesionales de la IS. Sin embargo, no es recomendable ir más allá del corto o medio plazo, precisamente por el dinamismo del contexto anteriormente citado. La preocupación por la evolución de los perfiles profesionales ha sido una constante en el ámbito académico y en el contexto profesional, generando en ocasiones tensiones entre ambos. Al igual que en otras disciplinas, el modelo formativo de la educación superior parece no satisfacer los deseos de empleabilidad inmediata del sector empresarial. Para valorar posibles perfiles profesionales que puedan ser demandados a corto, medio y largo plazo, pueden identificarse dos fuentes de información principales:

- La bibliografía académica: su contenido refleja los frentes de investigación y las innovaciones metodológicas y técnicas. En el campo de la información y documentación se dispone de un elevado volumen de estudios sobre las necesidades y el comportamiento de los usuarios de la información. Su estudio delinea los potenciales requerimientos a medio y largo plazo.

- Las ofertas y demandas de empleo: reflejan los requerimientos y necesidades a corto plazo del entorno socioeconómico y cultural. El estudio de diferentes aspectos del mercado laboral también ha sido constante en la disciplina.

Existe abundante bibliografía sobre el mercado laboral en el contexto español, y nuevos estudios van apareciendo regularmente, incluyendo trabajos recientes publicados en El profesional de la información y en Anuario ThinkEPI. Sin embargo, no se ha recurrido a ellos en este texto, en cuanto dibujan un panorama estático y puntual de la demanda laboral. Se ha preferido plantear una revisión selectiva de literatura académica sobre perfiles, que cubre los últimos 20 años. Gwyer (2015) ha adoptado un enfoque similar, realizando una revisión sistemática de literatura, y ha validado esta aproximación. Las conclusiones a las que llega son similares a las contempladas en este texto. No es el único precedente: Vassilakaki y Moniarou-Papaconstantinou (2015) han analizado la literatura especializada para identificar los roles que los bibliotecarios han desempeñado desde 2000, estableciendo la existencia de seis perfiles principales:

- profesores;

- especialistas en tecnología;

- bibliotecarios embebidos o incrustados;

- consultores de información;

- gestores de conocimiento;

bibliotecarios especializados.

\section{Romanos frente a cartagineses (disciplina versus profesión)}

Al abordar una reflexión sobre perfiles profesionales, resulta ineludible cuestionar la definición y los fundamentos de la disciplina o disciplinas que los sustentan. La IS sigue siendo objeto de reflexión y discusión en cuanto a su esencia, objetivos y objeto, y a sus relaciones con otras disciplinas. El dinamismo y el cambio que afectan al entorno profesional también influye sobre el mundo académico.

En un texto que teóricamente debería versar sobre "nuevos" perfiles profesionales, me voy a permitir retroceder veinte años, concretamente al discurso de aceptación del ACM SIGIR Gerard Salton Award for Excellence in Research que fue concedido a Tefko Saracevic en 1997. En el mismo, este investigador defendía que: 
"la ciencia de la información se ocupa de la práctica profesional y de la investigación científica relacionada con la comunicación efectiva y eficiente de los registros del conocimiento humano entre las personas, en el contexto de las necesidades de información individuales, organizativas y sociales, y en el uso de esa información",

para establecer que:

"el estudio apropiado para la ciencia de la información es el problema de la interfaz efectiva y eficiente entre las personas y la literaturas" (Saracevic, 1997, p. 20).

Saracevic entiende como "literaturas" los registros del conocimiento humano. Este párrafo puede ser aceptado como fundamento tanto por la disciplina científica como por el mundo del ejercicio profesional, e igualmente como base para la formulación de cualquier perfil profesional, independientemente de épocas, modas y tecnologías. Sin embargo, una definición aceptada, amplia y genérica no suele ser suficiente cuando se trata de trasladarla a acciones y procesos específicos.

La bibliografía sobre este tema incide, como puede verse en diferentes autores, sobre la dualidad entre ciencia y servicio. Buckland (2012) se planteaba que la ciencia de la información se centraba en hacer que la gente estuviese mejor informada y que, en consecuencia, era una forma de compromiso cultural, una ciencia de lo artificial. Recientemente Yu (2015) ha propuesto una refundación de los conceptos de dato, información, conocimiento y documento, con el objeto de establecer el fundamento teórico de la ciencia de la información y la documentación. Robinson y Bawden (2014) han realizado un estudio sobre los conceptos emergentes de información en varias disciplinas y sus relaciones con la ciencia de la información y la documentación. Si la discusión sobre los fundamentos es continua a nivel académico, puede suponerse que se reproduce en la definición del campo profesional.

\section{La táctica gana batallas, la estrategia gana la guerra (perfiles frente a competencias)}

En un interesante trabajo, Broady-Preston (2010) ha revisado la noción de "profesión" en el campo de la información. Su conclusión, con la que coincidimos plenamente, es que la profesión no debe ni puede definirse mediante un "qué es", sino a través de las competencias y habilidades de los profesionales. La reflexión sobre las competencias no es reciente. Moore (1998) ya señalaba cuatro conjuntos de competencias que los perfiles profesionales de los especialistas en información debían desempeñar, y que correspondían a:

- creación;

- colección y recopilación;

- comunicación;

- consolidación de información.

Griffiths (1998, p. 8) reflexionaba que los profesionales debían incorporar a sus competencias la resolución de problemas, el trabajo en equipo y el aprendizaje continuo a lo largo de la vida, y señalaba cinco características clave para cualquier perfil profesional de las ciencias de la información y la documentación:
- servir como guía a los recursos de información;

- habilidad para colaborar en equipos, grupos y comunidades;

- saber priorizar y ser flexibles en los objetivos y procesos;

- capacidad de empoderar a las personas;

- capacidad de comprender las competencias nucleares y básicas de la organización.

E introducía la importante idea de que iba a resultar difícil predecir cómo iba a evolucionar el campo, en virtud de las características del contexto social:

"Los profesionales de la información del futuro necesitan tener el espíritu del pionero. Tienen que estar dispuestos a aceptar que no podemos predecir lo que la gente va a hacer, cómo lo van a hacer, o con quién" (Griffiths, 1998, p. 12).

\section{La profesión no debe ni puede definirse} mediante un "qué es", sino a través de las competencias y habilidades de los profesionales

Desde la década de 2000 la necesidad de ser flexibles y adaptativos como profesionales ha estado presente en la bibliografía. Newman, Porter y Yang (2001) indicaban varias tendencias para esa década, y demandaban que el especialista en información fuese capaz de aprender a manejar nuevas, herramientas, interpretar los resultados, y transferirlos a los usuarios en forma comprensible y utilizable. En 2004 Ashcroft señalaba que se necesitaban habilidades de mercadotecnia, evaluación y adaptación a las tecnologías, capacidad de aprendizaje, y habilidades de comunicación, negociación y colaboración. Feather anunciaba que la gestión de la información estaba siendo ya abordada como una herramienta básica propia por otros profesionales de diferentes tipos y clases, y que los especialistas en información, especialmente los bibliotecarios, estaban dejando de ser imprescindibles en el entorno de los sistemas de información científica. $Y$ tras revisar las tendencias en el acceso y uso de la información, indicaba que:

"Esto crea un nuevo papel para el profesional de la información; ya no sólo se centra en el suministro y el análisis de la información, sino que en el rico entorno en información en el que todos vivimos también debe ser un instructor en cómo hacer el mejor uso de la información que está disponible y cómo evaluar lo encontrado" (Feather, 2006, p. 5).

Missingham (2006) describía cuatro competencias fundamentales para los profesionales del siglo XXI, que redactaba literariamente como:

- mountain climbing;

- jumping;

- orienteering;

- endurance.

Cada una de estas competencias se ilustraba con actividades y proyectos específicos dentro del ámbito de bibliotecas. Como puede deducirse de los términos usados, estaban 
relacionados con la capacidad de crear nuevos diseños, la innovación, la formación y la adaptación. Una década después, son competencias que se demandan continuamente como básicas para un buen profesional.

Si bien muchos trabajos de análisis se centran en los perfiles bibliotecarios, también es posible encontrar trabajos sobre los archiveros. Kallberg (2012) ha identificado la evolución tecnológica como un factor clave en las características y funciones de la actividad profesional del archivero para los próximos años. Junto a ello corre paralela la gestión de documentos electrónicos, y su preservación a largo plazo. Incluye además como funciones profesionales la auditoría de la información, y el control y acceso a la información por parte de los ciudadanos.

La primera afirmación que puede hacerse tras la revisión de estas fuentes es que, más que a nuevos perfiles profesionales, se está haciendo referencia a saberes y habilidades específicas que los profesionales deben atender e incorporar a su saber hacer, y que corresponderían a las tan traídas y llevadas competencias. No se estaría tratando entonces de nuevos perfiles profesionales propiamente dichos, sino de perfiles especializados, que se caracterizan por una combinación de competencias. Será necesario plantear la perduración o presencia y la variabilidad de estos perfiles en el ámbito profesional a corto y medio plazo.

Los profesionales de la información del futuro necesitan tener el espíritu del pionero, estar dispuestos a aceptar que no podemos predecir lo que la gente va a hacer, cómo lo van a hacer, o con quién

\section{Cambios de alianzas (la amenaza tecnológica)}

La influencia que las tecnologías de la información y la comunicación están ejerciendo, a corto y medio plazo, sobre los perfiles profesionales no ha pasado desapercibida a los propios profesionales. Hicks (2014) ha destacado que son conscientes de ello y que comprenden que esto está cambiando la sustancia de su trabajo, lo que afecta además a su propia percepción y a la de terceros, ya que la identidad profesional está evolucionando constantemente.

Ataman defiende que los futuros profesionales requieren una formación predominante en materias tecnológicas, proponiendo la integración en el campo de la ingeniería y señalando que:

"En este nuevo entorno ya no hay necesidad de profesionales de la información, tales como archiveros, bibliotecarios y gestores de registros, en su papel tradicional de 'personas que ayudan en el acceso a la información'" (Ataman, 2009, p. 217).

Este investigador propone cuatro grandes áreas profesionales en las que desempeñar una actividad especializada en el entorno digital, correspondientes a:

- presentación y etiquetado de información, su almacenamiento confiable;
- tratamiento "forense" (en el sentido de ser capaz, si es necesario, de descubrir, trazar y analizar el ciclo de vida de la información),

- conservación;

- gestión de documentos (records management).

Carroll, ante la "amenaza" tecnológica, se preguntaba sobre el papel de los profesionales:

"Y si vamos a un futuro donde todo lo que tiene que hacer el que busca información información es preguntar a una 'máquina', entonces ¿cuál es el papel del profesional de la información?" (Carroll, 2012, p. 173).

Y como respuesta plantea cuatro desafíos claros para el profesional:

1) cambiar rápidamente los servicios a demanda del usuario;

2) respuestas para la sobrecarga de información;

3) globalización del conocimiento;

4) cambios culturales en el mundo digital, en especial sobre propiedad intelectual y privacidad.

Cabe plantear sin embargo, si la importancia del componente tecnológico parece estar disminuyendo, al menos formalmente. Un reciente estudio (Thelwall; Maflahi, 2015) llama la atención sobre cómo ha ido disminuyendo progresivamente durante la última década la presencia de términos tecnológicos en la literatura especializada sobre LIS (library and information science). Sin estar de acuerdo por completo con sus conclusiones, sí que cabe destacar que la interiorización de la tecnología ha favorecido que los practicantes de la IS pongan el foco de atención en nuevas competencias que complementan la formación tradicional, y que le aporten valor añadido como profesionales. La tecnología es un factor muy influyente, al igual que en muchos otros aspectos. Tendemos a hablar del impacto de la tecnología, cuando en realidad deberíamos pensar en los cambios en el medio y el contexto social, económico y cultural en toda su amplitud. En cualquier caso, va a ser necesario aceptar, parafraseando libremente a Arthur C. Clarke, que

"cualquier profesional que pueda ser sustituido por una máquina, será sustituido por una máquina".

\section{Todos los caminos conducen a Roma}

"Ahora las $i$-Schools preparan una amplia gama de profesionales de la información, incluyendo arquitecto de información, analista de datos, administrador de base de datos, desarrollador web, ontólogo, ingeniero de usabilidad, estratega de medios sociales, curador de datos, director o responsable de información y, por supuesto, bibliotecario, archivero y curador de museo. Esta gama hace que sea más difícil prever la preparación necesaria para los futuros especialistas en información, ya que los estudiantes vienen a nuestra escuela a prepararse para muy diversas carreras. Agrava la dificultad el hecho de que es imposible predecir el entorno de información donde nuestros graduados van a trabajar en la próxima década" (Moran; Marchionini, 2012, p. 96).

La revisión de la bibliografía y de los medios sociales del campo de la IS permite avanzar un buen número de teóricos perfiles: 
- bibliotecas y colecciones digitales;

- bibliotecario de datos;

- bibliotecario embebido;

- estudios de necesidades de información y comportamiento informacional;

- cloud computing;

- metadatos;

- ontologías;

- alfabetización informacional;

- formación;

- arquitecto de información...

Se ha señalado la implicación de los bibliotecarios en el gobierno electrónico, a través de programas de información y de formación (Jaeger et al., 2012). También se ha destacado la integración en los procesos de investigación llevados a cabo en las instituciones académicas (Schonfeld, 2012), así como los desafíos que plantea la gestión de los datos de investigación (Jørn-Nielsen; Hjørland, 2014). La inteligencia competitiva es otro de los campos en expansión (Jin; Ju, 2014). Tampoco pasan desapercibidas las posibilidades en áreas de acción específicas, como la ayuda al desarrollo y la preocupación ambiental (Abiolu; Okere, 2012).

Pero no se trata sólo de actividades y procesos. El propio objeto documental influye en esos perfiles. Gradmann (2014) ha destacado el cambio de paradigma que supone la transición desde la noción de documento, en el sentido tradicional y como objeto de trabajo de la profesión desde sus inicios, hacia un entorno dinámico, distribuido e hipertextual basado en el etiquetado semántico (linked data).

Se trata de haber dotado al futuro profesional de las competencias necesarias, y de los mecanismos de adaptación suficientes, para que pueda aplicar su saber hacer en cualquier entorno de actividad

\section{Delenda est Carthago?}

“Una profesión desaparece sólo cuando su misión se convierte en irrelevante para la sociedad o todas las actividades requeridas para su misión puede ser automatizadas" (Yu, 2015, p. 810).

No parece que éste sea el destino de los practicantes de la IS, al menos a corto y medio plazo. El desarrollo de perfiles profesionales adecuados sólo puede provenir del estudio sobre la práctica y su combinación con el análisis de los frentes de investigación, que deben reflejarse en los programas educativos y formativos que se lleven a cabo en las instituciones de enseñanza superior. No se trata entonces de mirar los títulos de los perfiles, lo que puede suponer una aproximación superficial. Lo verdaderamente interesante es abordar los contenidos que tiene ese perfil, qué se le puede demandar y qué debe saber hacer. $Y$ por supuesto, se trata de haber dotado al futuro profesional de las competencias necesarias, y de los mecanismos de adaptación suficientes para que pueda aplicar su saber hacer en cualquier entorno de actividad.
Una preocupación relacionada con los perfiles profesionales es la pretendida invisibilidad de la profesión. Esta invisibilidad del perfil del profesional de la información no es nueva. Saracevic (1997, p. 25) ya destacaba que muchos esfuerzos en otras áreas estaban dando como resultado la reinvención de la rueda, por el desconocimiento de la ciencia de la información y de su experiencia y actividad profesional, algo muy similar a lo que podemos ver en la actualidad.

Curiosamente, frente a la preocupación por el posicionamiento en el campo profesional, las motivaciones para estudiar IS no parecen coincidir con las preocupaciones de los profesionales y los académicos. Un reciente estudio (Moniarou-Papaconstantinou; Vassilakaki; Tsatsaroni, 2015) revela que la titulación es elegida principalmente por apego al libro, a la lectura y el trabajo bibliotecario, y como una segunda carrera por profesionales que ven en ella la posibilidad de usar en su actividad y trayectoria el conocimiento y las competencias que se adquieren.

La vigilancia del entorno y los replanteamientos profesionales deben ser un proceso continuo

Las amenazas a la profesión y a su actividad resultan ser, al mismo tiempo, un buen conjunto de oportunidades (Law, 2014). La IS se encuentra en un entorno cambiante, que exige adaptarse rápidamente. En el contexto de la sociedad del conocimiento, dispone de las habilidades y competencias más importantes para triunfar: las relacionadas con la gestión de información. Sin embargo, la vigilancia del entorno y los replanteamientos profesionales deben ser un proceso continuo. La IS no puede arriesgarse a perder: Aníbal ganó famosas batallas y pasó a la historia, pero Cartago acabó perdiendo la guerra. Tito Livio $(22,51)$ puso una sentencia demoledora en boca de Maharbal, cuando éste vio que Aníbal no se decidía a tomar Roma: "Sabes vencer, Aníbal, pero no sabes qué hacer con la victoria".

\section{Bibliografía}

Abiolu, Oluremi A.; Okere, Oluchi O. (2012). “Environmental literacy and the emerging roles of information professionals in developing economies". IFLA Journal, v. 38, n. 1, pp. 53-59. http://conference.ifla.org/past-wlic/2011/109-abiolu-en.pdf http://dx.doi.org/10.1177/0340035211435070

Ashcroft, Linda (2004). "Developing competencies, critical analysis and personal transferable skills in future information professionals". Library review, v. 53, n. 2, pp. 82-88.

http://dx.doi.org/10.1108/00242530410522569

Ataman, Bekir-Kemal (2009). "Requirements for information professionals in a digital environment: Some thoughts". Program: Electronic library \& information systems, v. 43, n. 2, pp. 215-228.

http://dx.doi.org/10.1108/00330330910954415

Broady-Preston, Judith (2010). "The information professional of the future: Polymath or dinosaur?". Library management, v. 31, n. 1/2, pp. 66-78.

http://dx.doi.org/10.1108/01435121011013412 
Buckland, Michael (2012). "What kind of science can information science be?". Journal of the American Society for Information Science and Technology, v. 63, n. 1, pp. 1-7. http://people.ischool.berkeley.edu/ buckland/whatsci.pdf http://dx.doi.org/10.1002/asi.21656

Carroll, Bonnie C. (2012). "From knowledge navigator and Watson to Star Trek: The role of the information professional". Information services \& use, v. 32, n. 2-3, pp. 171-176. http://dx.doi.org/10.3233/ISU-2012-0667

Feather, John (2006). "The context of change: Information professionals and the information professions in an information society". Health information and libraries journal, v. 23, supl. 1, pp. 3-9.

http://dx.doi.org/10.1111/j.1471-1842.2006.00685.x

Gradmann, Stefan (2014). "From containers to content to context". Journal of documentation, v. 70, n. 2, pp. 241-260. http://dx.doi.org/10.1108/jd-05-2013-0058

Griffiths, Jose-Marie (1998). "The new information professional". Bulletin of the American Society for Information Science and Technology, v. 24, n. 3, pp. 8-12.

http://dx.doi.org/10.1002/bult.83

Gwyer, Roisin (2015). "Identifying and exploring future trends impacting on academic libraries: A mixed methodology using journal content analysis, focus groups and trend reports". New review of academic librarianship, v. 21, p. 3, pp. 269-285.

http://dx.doi.org/10.1080/13614533.2015.1026452

Hicks, Deborah (2014). "Information technology, change and information professionals' identity construction: A discourse analysis". Proceedings of the American Society for Information Science and Technology, v. 51, n. 1, pp. 1-9. http://dx.doi.org/10.1002/meet.2014.14505101049

Jaeger, Paul T.; Bertot, John-Carlo; Shuler, John A.; McGilvray, Jessica (2012). "A new frontier for LIS programs: E-government education, library/government partnerships, and the preparation of future information professionals". Education for information, v. 29, n. 1, pp. 39-52.

http://dx.doi.org/10.3233/EFI-2010-0893

Jin, Tao; Ju, Boryung (2014). "Towards understanding the perceptions of information professionals about competitive intelligence work". Journal of information \& knowledge management, v. 13, n. 2.

http://dx.doi.org/10.1142/s0219649214500117

Jørn-Nielsen, Hans; Hjørland, Birger (2014). “Curating research data: The potential roles of libraries and information professionals". Journal of documentation, v. 70, n. 2, pp. 221-240.

http://dx.doi.org/10.1108/jd-03-2013-0034

Kallberg, Maria (2012). "Archivists 2.0: Redefining the archivist's profession in the digital age". Records management journal, v. 22, n. 2, pp. 98-115.

http://dx.doi.org/10.1108/09565691211268162

Law, Derek (2014). "The world is our lobster". New library world, v. 115, n. 5/6, pp. 200-210.

http://dx.doi.org/10.1108/n/w-05-2014-0054
Missingham, Roxanne (2006). "Library and information science: Skills for twenty-first century professionals". Library management, v. 27, n. 4/5, pp. 257-268.

http://dx.doi.org/10.1108/01435120610668197

Moniarou-Papaconstantinou, Valentini; Vassilakaki, Evgenia; Tsatsaroni, Anna (2015). "Choice of library and information science in a rapidly changing information landscape". Library management, v. 36, n. 8/9, pp. 584-608. http://dx.doi.org/10.1108/Im-04-2015-0022

Moore, Nick (1998). "The future demand for information professionals in Europe". Education for information, v. 16, n. 3, pp. 191-208.

http://dx.doi.org/10.3233/EFI-1998-16301

Moran, Barbara B.; Marchionini, Gary (2012). "Information professionals 2050: Educating the next generation of information professionals". Information services \& use, v. 32, n. 3-4, pp. 95-100.

http://dx.doi.org/10.3233/ISU-2012-0674

Newman, Nils C.; Porter, Alan L.; Yang, Julie (2001). "Information professionals: Changing tools, changing roles". Information outlook, v. 5, n. 3, pp. 24.

http://units.sla.org/division/dpht/meetings/spring2010/ PHTD2010-hawes.pdf

Robinson, Lyn; Bawden, David (2014). "Mind the gap: Transitions between concepts of information in varied domains". En: Ibekwe-SanJuan, Fidelia; Dousa, Thomas M. Theories of information, communication and knowledge. A multidisciplinary approach. Springer Verlag, pp. 121-141. ISBN: 9789400769724 http://dx.doi.org/10.1007/978-94-007-6973-1_6

Rowlands, lan; Nicholas, David; Williams, Peter; Huntington, Paul; Fieldhouse, Maggie; Gunter, Barrie; Withey, Richard; Jamali, Hamid R.; Dobrowolski, Tom; Tenopir, Carol (2008). "The Google generation: The information behaviour of the researcher of the future". Aslib proceedings, v. 60, n. 4, pp. 290-310. http://late-dpedago.urv.cat/site_media/papers/425.pdf http://dx.doi.org/10.1108/00012530810887953

Saracevic, Tefko (1997). "Users lost: reflections on the past, future, and limits of information science". ACM SIGIR Forum, v. 31, n. 2, pp. 16-27.

$h t t p: / / d x . d o i . o r g / 10.1145 / 270886.270889$

Schonfeld, Roger (2012). "Information professionals to serve academia." Information services \& use, v. 32, n. 3-4, pp. 227-229. http://dx.doi.org/10.3233/ISU-2012-0680

Thelwall, Mike; Maflahi, Nabeil (2015). "How important is computing technology for library and information science research?". Library \& information science research, v. 37, n. 1, pp. 42-50. http://dx.doi.org/10.1016/j.lisr.2014.09.002

Vassilakaki, Evgenia; Moniarou-Papaconstantinou, Valentini (2015). "A systematic literature review informing library and information professionals' emerging roles". New library world, v. 116, n. 1/2, pp. 37-66.

http://dx.doi.org/10.1108/n/w-05-2014-0060

Yu, Liangzhi (2015). "Back to the fundamentals again". Journal of documentation, v. 71, n. 4, pp. 795-816. http://dx.doi.org/10.1108/jd-12-2014-0171 